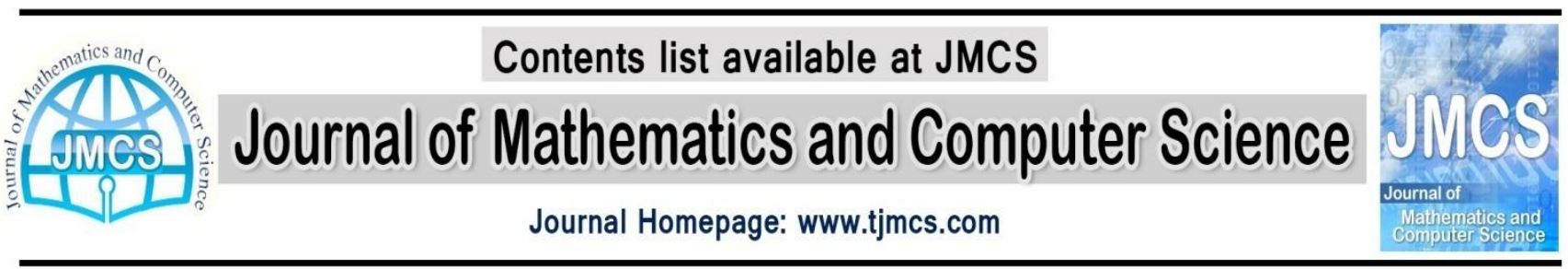

\section{Comparison of Fuzzy Numbers with Ranking Fuzzy and Real Number}

\author{
M. Yaghobi ${ }^{1}$, M. Rabbani ${ }^{2 *}$, M. Adabitabar Firozja ${ }^{3}$ J. Vahidi $^{4}$ \\ ${ }^{1}$ Department of Mathematics, Mazandaran Branch Science and Research, Islamic Azad University, \\ Mazandaran, Iran. \\ $2^{*}$ Department of Mathematics Sari Branch, Islamic Azad University, Sari, Iran. \\ mrabbani@iust.ac.ir \\ ${ }^{3}$ Department of mathematics, Qaemshar Branch, Islamic Azad University, Qaemshahr, Iran. \\ ${ }^{4}$ Department of Applied Mathematics, Iran University of Science and Technology, Behshahr, Iran, \\ jvahidi@iust.ac.ir
}

Article history:

Received May 2014

Accepted July 2014

Available online August 2014

\begin{abstract}
Ranking fuzzy numbers play as a key tool in many applied models in the world and in particular decision-making procedures. We are going to present a new method based on the ranking the fuzzy number and real number. The problem of ranking the fuzzy number and real number is proposed with ranking function and then this approach to extend the ranking of two fuzzy numbers with ranking function. The proposed method is illustrated by some numerical examples and in particular the results of ranking by the proposed method and some common and existing methods for ranking fuzzy sets is compared to verify the advantage of the new approach. We will see that against of most existing ranking approaches where for two fuzzy sets are the exact ranking, the above men sioned method can give a ranking fuzzy numbers with acceptance rate smaller as fuzzy.
\end{abstract}

Keywords: Fuzzy numbers, Ranking.

\section{1 -Introduction}

Fuzzy ranking is a topic which has been studied by many researchers. In [6], Wang and Kree introduced reasonable properties for the ordering of fuzzy quantities .In [18], Abbasbandy and Asady defined a sign distance of fuzzy numbers and proposed a ranking method with a fuzzy origin for fuzzy numbers and distance of fuzzy numbers with respect to it' $\mathrm{s}$ origin. In [23], Yao and $\mathrm{Wu}$ defined a sign distance of fuzzy numbers and proposed a ranking method. In [20], Allah- viranloo and Adabitabar Firozja introduced a new metric distance on fuzzy numbers which was used for ranking fuzzy numbers by comparing with two crisp numbers max and min. Tran and Duckste in [15], compared the fuzzy numbers using a fuzzy distance measure. Some researchers for ranking fuzzy numbers introduced a defuzzification methods in [16, 13]. Fortemps and Roubens in [1], proposed a ranking method based on area compensation. Some of the other researchers have proposed functions for ranking. In Modarres and in [2], Sadi-Nezhad method a fuzzy number is evaluated by a function called preference function and ranked by preference ratio. Wang et al. In [22], 
defined the maximal and minimal reference sets of fuzzy numbers to measure LR deviation degree and then the transfer coecient was defined to measure the relative variation of LR deviation degree of fuzzy number and then proposed the ranking method based on the LR deviation degree and relative variation of fuzzy numbers. In [20], Asady proposed a revised method of ranking LR fuzzy number based on deviation degree with Wang's method. In [21], Wang and Luo presented a ranking approach for fuzzy numbers called area ranking based on positive and negative ideal points, which defined two indices for the purpose of ranking. In [19], Abbasbandy and Hajjari introduced a ranking of trapezoidal fuzzy numbers based on the left and the right spreads at some $\alpha$-levels of trapezoidal fuzzy numbers. Each method has a shortcoming. Adabitabar firozja et al. In [11], proposed a ranking function for ranking real numbers and fuzzy number with acceptance rate and then to extended for ranking two fuzzy numbers. In this paper, we proposed a new ranking function for ranking real numbers and fuzzy number with acceptance rate and then to extended for ranking two fuzzy numbers. The paper is organized as follows: The background on fuzzy concepts is presented in section 2. A comparison between one real number and a fuzzy number with its properties is introduced in Section 3. Subsequently, in Section 4 ranking of two fuzzy numbers and its properties is considered, Numerical Examples in section 5, finally, conclusion are drawn in Section 6.

\section{2 - Background}

A fuzzy set $\tilde{A}$ is a generalized left right fuzzy numbers (GLRFN) defined by Dubois and Prade[8], and denoted as $\tilde{A}=\left(a_{1}, a_{2}, a_{3}, a_{4}\right)_{L R}$, if it 's membership function satisfies the following:

$$
\mu_{\tilde{A}}(x)=\left\{\begin{array}{cc}
L\left(\frac{a_{2}-x}{a_{2}-a_{1}}\right), & a_{1} \leq x \leq a_{2} \\
1 & a_{2} \leq x \leq a_{3} \\
R\left(\frac{x-a_{2}}{a_{4}-a_{3}}\right), & a_{3} \leq x \leq a_{4}
\end{array}\right.
$$

Where $\mathrm{L}$ and $\mathrm{R}$ are strictly decreasing functions defined on $[0,1]$ and satisfying the conditions:

$$
\begin{aligned}
& L(t)=R(t)=1 \quad \text { if } t \leq 0 \\
& L(t)=R(t)=0 \quad \text { if } t>0
\end{aligned}
$$

Trapeziodal fuzzy numbers ( $\operatorname{TrFN}$ ) are special cases of GLRFN with $L(t)=R(t)=1-t$. A $\alpha$-level interval of fuzzy number $\tilde{A}$ is denoted as:

$$
[\tilde{A}]_{\alpha}=\left[A_{L}(\alpha), A_{u}(\alpha)\right]=\left[a_{2}-\left(a_{2}-a_{1}\right) L^{-1}(\alpha), a_{3}+\left(a_{4}-a_{3}\right) R^{-1}(\alpha)\right]
$$

And if $\lambda \in R$ then

$$
\begin{aligned}
& \tilde{A}+\lambda=\left(a_{1}+\lambda, a_{2}+\lambda_{a_{3}}+\lambda, a_{4}+\lambda\right)_{L R} \\
& \lambda \tilde{A}= \begin{cases}\left(\lambda_{a_{1}}, \lambda_{a_{2}}, \lambda_{a_{3}}, \lambda_{a_{4}}\right)_{L R}, & \lambda \geq 0 \\
\left(\lambda_{a_{4}}, \lambda_{a_{3}}, \lambda_{a_{2}}, \lambda_{a_{1}}\right)_{L R}, & \lambda<0\end{cases}
\end{aligned}
$$

We denote by $F_{L R}$ the set of generalized left right fuzzy numbers. 


\section{3 - Comparison between crisp and fuzzy number}

Let $\tilde{A} \in F_{L R}$ and $x \in R$ then we consider the following problem:

What do we say about whether $x$ is greater to $\widetilde{A}$ ?

To solve this problem, Adabitabar frozja et al. In [3] proposed a ranking function for ranking of fuzzy numbers and real numbers but, for two real numbers $\mathrm{x}, \mathrm{y}$ where:

$$
x, y \in\left[a_{2}-\left(a_{2}-a_{1}\right) L^{-1}\left(\frac{1}{2}\right), a_{3}-\left(a_{4}-a_{3}\right)_{R}^{-1}\left(\frac{1}{2}\right)\right] .
$$

If $x<y$ then $\tilde{A} \approx x$ and $\tilde{A} \approx y$, where this is not a good result.

In this paper, we proposed new ranking functions for comparison between crisp and fuzzy number. We extend the natural ordering relation $\leq$ on real numbers by increasing real function $L(\tilde{A},):. R \rightarrow[0,1]$, and $L(\widetilde{B},):. R \rightarrow[0,1]$, decreasing real function to introduce the ordering relation crisp and fuzzy number with the characteristic functions $L(\tilde{A}, x)$, (acceptance rate larger $\mathrm{x}$ of $\tilde{A})$ and $L(\tilde{B}, x)$, (acceptance rate larger $\mathrm{x}$ of $\tilde{B}$ ) as follow:

$$
L(A, x)=\frac{\int_{-\infty}^{x} \mu_{A}(t) d t}{\int_{-\infty}^{+\infty} \mu_{A}(t) d t}, \quad L(B, x)=\frac{\int_{-\infty \mu_{B}}^{x}(t) d t}{\int_{-\infty}^{+\infty} \mu_{B}(t) d t}
$$

Where with $\tilde{A}=(0,1,2,3)_{L R}$, Figure 1 shows the diagram of $L(\tilde{A}, x), L(x, \vec{A})$.
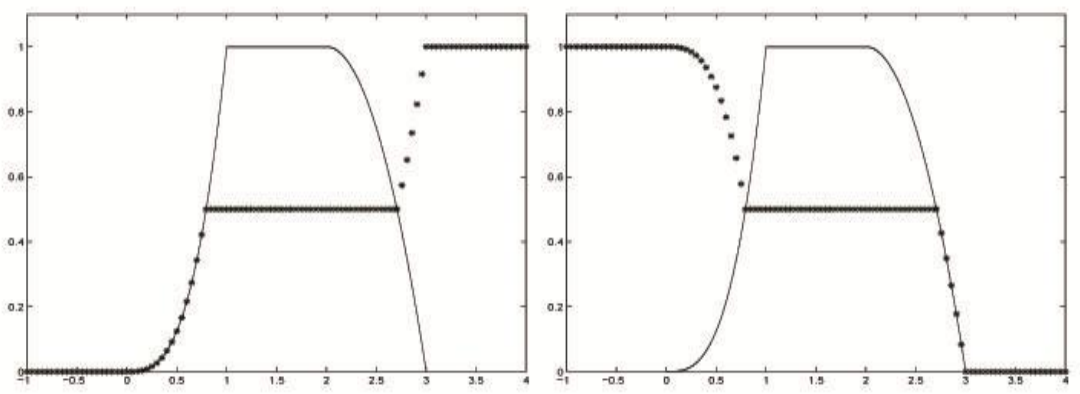

Figure 1: $\tilde{A}=(0,1,2,3)_{L R}-$ and $L(\tilde{A}, x) * * *$ and $L(x, \tilde{A}) * * *$.

\section{1- Some properties}

Let $\tilde{A} \in F_{L R}$ and $K \in R$, then

Proposition 1.

$$
L(k \tilde{A}, k x)=\left\{\begin{array}{ll}
L(\tilde{A}, x), & 0 \leq k \\
L(x, \tilde{A}), & k>0
\end{array}, L(k \tilde{B}, k x)= \begin{cases}L(\tilde{B}, x) & 0 \leq k \\
L(x, \tilde{B}) & k>0\end{cases}\right.
$$


Proposition 2. $L(\tilde{A}+x, x+k)=L(\tilde{A}, x), \quad L(\tilde{B}+x, x+k)=L(\tilde{B}, x) \quad k \in R$

Proposition 3.If $x<y$ then

$$
L(\tilde{A}, x)<L(\tilde{A}, y), \quad L(x, \tilde{A})>L(y, \tilde{A})
$$

Regarding to equations (5) proofs is evident.

\section{4- Ranking of two fuzzy numbers}

Let $\tilde{A} \in F_{L R}$ and $\widetilde{B} \in F_{L R}$ we consider the following problem:

What do we say about whether $\widetilde{A}$ is greater (smaller) than or equal to $\widetilde{B}$ ?

In order to solve this problem, Adabitabar frozja et al. [3] proposed a ranking function for ranking of two fuzzy numbers but in this paper we proposed the new ranking function for ranking of two fuzzy numbers. We have used ordering relations $L(\tilde{A}, x)$ and $L(\tilde{B}, x)$ where we defined in (6) and denoted ordering relation $\tilde{A}$ and $\tilde{B}$ by $R(\tilde{A}, \tilde{B})$ and defined such as follow:

$$
R(\tilde{A}, \tilde{B})=\underset{\operatorname{supp}(A \bigcup \operatorname{supp}(B)}{\int L(\tilde{A}, x)-L(\tilde{B}, x) d x}
$$

Where $L(\tilde{A}, x)$ defined fin equations $(6)$ and $L(\tilde{A}, \tilde{B})$ is acceptance

rate smaller $\tilde{A}$ of $x$. In this paper, to compare of two fuzzy numbers $\tilde{A}$ and $\tilde{B}$ all values in $\operatorname{Supp}(A, B)$ with the membership degree compare with $\tilde{A}$ and vice versa.

\section{1- Some properties}

For $\tilde{A}$ and $\tilde{B} \in F_{L R}$ and $k \in R$

Proposition 4. $R(A, A)=O$

Proof: Recording to equation (10).

$$
R(\tilde{A}, \tilde{A})=\underset{\operatorname{supp}(A) \bigcup \operatorname{supp}(A)}{\int(L(\tilde{A}, x)-L(\tilde{A}, x)) d x=0}
$$

Proposition 5. $R(A, B)=-R(B, A)$

Proof: Recording to equation (10)

$$
\begin{aligned}
(\tilde{A}, \tilde{B})= & \int L(A, x)-L(B, x) d x \\
= & \operatorname{supp}(A) \cup \operatorname{supp}(B) \\
& \int L(B, x)-L(A, x) d x=-R(\tilde{A}, \tilde{B})
\end{aligned}
$$


Proposition 6: $R(\tilde{A}+k, \tilde{B}+k)=R(\tilde{A}, \tilde{B}), \quad k \in R$

Proof: By considering to equations (8) and (10).

$$
R(\tilde{A}+k, \tilde{B}+k)=\begin{aligned}
& \int(\tilde{A}+k, \tilde{B}+k) d x \\
& \operatorname{supp}(\tilde{A}+k) \cup \operatorname{supp}(\tilde{B}+k)
\end{aligned}
$$

If $x=k+t$ then $\quad d x=d t, \mathrm{~A}$ cording to $\mathrm{t}=\operatorname{supp}(A) \cup \operatorname{supp}(B), \mathrm{X}=\operatorname{supp}(A+k) \cup \operatorname{supp}(B+k)$

we have :

$$
\begin{aligned}
& =\int_{\operatorname{supp}(A+k) \bigcup \operatorname{supp}(B+k)}\left(\frac{\int_{-\infty}^{t+k} \mu_{A+k}(t+k) d t}{\int_{-\infty}^{+\infty} \mu_{A+k}(t+k) d t}-\frac{\int_{-\infty}^{t+k} \mu_{B+k}(t+k) d t}{\int_{-\infty}^{+\infty} \mu_{B+k}(t+k) d t}\right) d(t+k) \\
& =\operatorname{supp}(A) \operatorname{supp}(B) \\
& \left.\int_{-\infty}^{t} \int_{-\infty \mu_{A}(t) d t}^{+\infty} \mu_{A}^{(t) d t}-\frac{\int_{-\infty}^{t} \mu_{B}(t) d t}{\int_{-\infty}^{+\infty} \mu_{B}(t) d t}\right) d(t)==R(\tilde{A}, \tilde{B})
\end{aligned}
$$

Proposition 7: $R(k A, k A)= \begin{cases}R(A, B) & k \geq 0 \\ -R(A, B) & k<0\end{cases}$

Proof: From equations (8), (10) and also $k \geq 0, k \in R$ we can write

$$
R(k A, k B)=\int_{\operatorname{supp}(k A) \cup \operatorname{supp}(k B)} \frac{\int_{-\infty}^{x} \mu_{k A}(x) d x}{\int_{-\infty}^{+\infty} \mu_{k A}(x) d x}-\frac{\int_{-\infty}^{x} \mu_{k B}(x) d x}{\int_{-\infty}^{+\infty} \mu_{k B}(x) d x} d x
$$

According to $\quad t=\operatorname{supp}(A) \cup \operatorname{supp}(B), x=\operatorname{supp}(k A) \cup \operatorname{supp}(k B), \quad$ we have:

$$
=\int_{\operatorname{supp}}\left(k A \bigcup \operatorname{supp}(k B) \int_{\int_{-\infty}^{+\infty} \mu_{k A}(k t) d(k t)}^{\int_{k} t}-\frac{\int_{-\infty}^{k t} \mu_{k B}(k t) d(k t)}{\int_{-\infty}^{+\infty} \mu_{k B}(k t) d(k t)} d t=R(A, B)\right.
$$

Definition 1.We define the ranking of $\tilde{A} \in F_{L R}, \tilde{B} \in F_{L R}$ and $x \in R$ by $L(\tilde{A}, x), L(\tilde{B}, x)$ :

1) If $R(\tilde{A}, \tilde{B})<0 \leftrightarrow \tilde{A} \geq \tilde{B}$

2) If $R(\tilde{A}, \tilde{B})=0 \leftrightarrow \tilde{A} \approx \tilde{B}$

3) If $R(\tilde{A}, \tilde{B})>0 \leftrightarrow \tilde{A} \leq \tilde{B}$

\section{Numerical Examples}


We have considered some examples constructed and discussed in $[4,16]$ for comparing the current method with some other ranking methods, where results of some other ranking rules [4] are shown in Table 1 and results of current method are shown the following form:

Set1: $A_{1}=(0.4,0.9,1), A_{2}=(0.4,0.5,1), A_{3}=(0.4,0.5,1)$ then $R\left(A_{1}, A_{2}\right)=-0.066$,

$R\left(A_{2}, A_{3}\right)=-0.066$ and $R\left(A_{1}, A_{3}\right)=-0.133$ therefore, $A_{1} \geq A_{2}, A_{2} \geq A_{3}, A_{1} \geq A_{3}$.

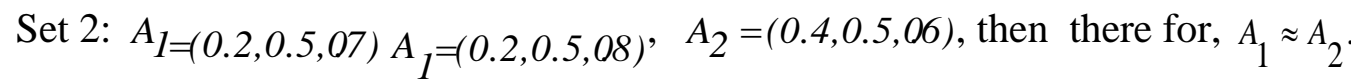

Set $\quad 3: A_{1}=(0.5,0.7,09), \quad A_{2}=(0.3,0.7,09) \quad, A_{3}=(0.3,0.4,07,0.9) \quad$ so, $\quad R\left(A_{1}, A_{2}\right)=-0.066$, $R\left(A_{2}, A_{3}\right)=-0.214$ and $R\left(A_{1}, A_{3}\right)=-0.148$, therefore, $A_{1} \geq A_{2}, A_{2} \geq A_{3}, A_{1} \geq A_{3}$.

The proposed method has been used by continuous function for ranking and gives the acceptance rate.

\section{6 -Conclusions}

In this paper, initially we proposed a ranking approach for ranking one crisp real number and one fuzzy number with membership function then have extended this approach for ranking two fuzzy numbers. For comparing of two fuzzy numbers $\tilde{A}$ and $\widetilde{B}$ on $\operatorname{Supp}(\tilde{A})$ to the membership degree, is compared with $\widetilde{B}$ also, on $\operatorname{Supp}(\widetilde{B})$ with the membership degree, is compared with $\tilde{A}$. Thus, we obtain some useful properties. Some numerical examples have been presented in order to compare the proposed method of ranking with some of the approaches. This method was used a continuous function for ranking and gives the acceptance rate.

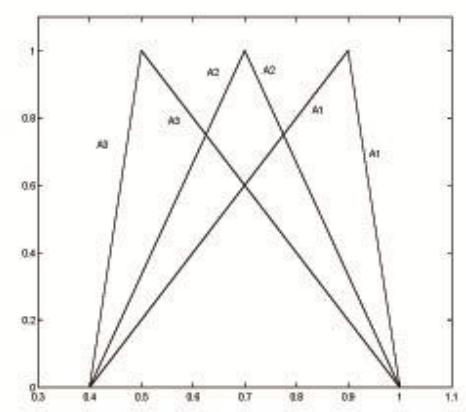

Figure 2: Set 1- $\left\{\tilde{A}_{1}, \tilde{A}_{2}, \tilde{A}_{3}\right\}$

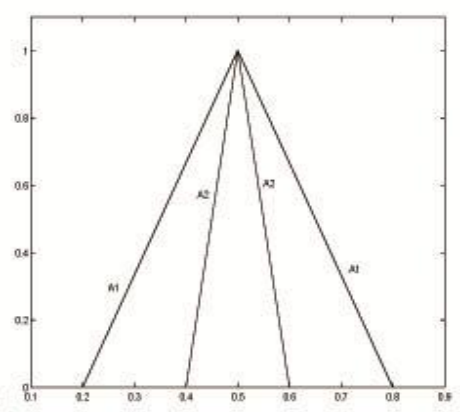

Set 2- $\left\{\tilde{A}_{1}, \tilde{A}_{2}, \tilde{A}_{3}\right\}$

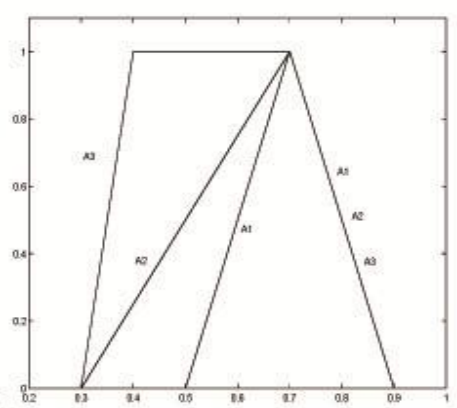

Set 3- $\left\{\tilde{A}_{1}, \tilde{A}_{2}, \tilde{A}_{3}\right\}$

\section{References:}

[1] A. Esi, On some new paranormed sequence spaces of fuzzy numbers de_nedby Orlicz functions and statistical convergence, Math. Modelling Analysis,4(2006), 379-388.

[2] A. Mahmodi Nejad and M. Mashinchi, Ranking fuzzy numbers based on the areas on the left and the right sides of fuzzy number, Computers and Mathematics with Applications 61 (2011), 431-442.

[3] B. Asady, The revised method of ranking LR fuzzy number based on deviation degree, Expert Systems with Application 37 (2010), 5056-5060.

[4] B.C. Tripathy and A. Baruah, Lacunary statistically convergent and lacunary strongly convergent generalized difference sequences of fuzzy real numbers, Kyungpook Mathematical Journal, 50(2010), 565-574.

[5] B.C. Tripathy and A. J. Dutta : On I-acceleration convergence of sequencesof fuzzy real numbers, Mathematical Modelling and Analysis,17(2012),549-557.

[6] B.C. Tripathy and B. Sarma : On I-convergent double sequences of fuzzy real numbers; Kyungpook Mathematical Journal, 52(2012), 189-200. 
[7] C.H. Cheng, A new approach for ranking fuzzy numbers by distance method, Fuzzy sets and Systems 95 (1998), 307-317.

[8] D. Dubios and H. prade, Fuzzy sets and Systems: Theory and Application,Academic Press, New york, 1980.

[9] G. Bortolan and R. Degan, A review of some method for ranking fuzzysets, fuzzy sets and Systems 15 (1985), 1-19.

[10] L. Tran and L. Duckstein, Comparison of fuzzy numbers using a fuzzydistance measure, Fuzzy Sets and Systems 130 (2002), 331-341.

[11] M. Adabitabar Firozja, B.Agheli and M. Hosseinzadeh, Ranking function of two LR-fuzzy numbers, Journal of Intelligent and Fuzzy Systems (2013), accepted.

[12]. M. Adabitabar Firozja, T. Houlari, Comparison of fuzzy numbers with ranking function of two LR-fuzzy numbers

[13] M,Ma, A. Kandel and M. Friedman,A new approach for defuzzification, Fuzzy Sets and Systems 111 (2000),351-356.6

[14] M. Ma, A. Kandel and M. Friedman, Correction to "A new approach for defuzzification", Fuzzy Sets and Systems 128 (2000), 133-134.

[15] M. Modarres, S.S. Nezhad, Ranking fuzzy numbers by preference ratio, Fuzzy Sets and Systems 118 (2001), 429-439.

[16] O. Kaleva and S. Seikkala: On fuzzy metric spaces, Fuzzy Sets Systems,12(1984), 215-229.

[17] P. Fortemps and M. Roubens, Ranking and defuzziifcation method based on area compensation, Fuzzy Sts and Systems 82 (1996), 319-330.

[18] S. Abbasbandy and B. Asady, Ranking of Fuzzy numbers by sign distance, Information Sciences 176 (2006), 2405-2416.

[19] S. Abbasbandy and T. Hajjari, A new approach for ranking of trapezoidal fuzzy numbers, Computing and Methematics with Application 57 (2009),413-419.

[20] T. Allahviranloo and M. Adabitabar Firozja, Ranking of fuzzy numbers bya new metric, Soft Computing14 (2010),773-782.

[21] X.Wang and E.E. Kree, Reasonable Properties for the ordering of fuzzy quantities I, Fuzzy Sets Systems, 118 (2001),375-385.

[22] Y.M. Wang and Y. Luo, Area ranking of fuzzy numbers based on positiveand negative ideal point, Computers and Mathematics with Applications58 (2009), 1769-1779.

[23] Z.X. Wang, Y.J. Liu, Z.P. Fan and B. Feng, Ranking LR fuzzy number based on deviation degree, Information Sciences 179 (2009), 2070-2077.

Table 1: Comparison on fuzzy numbers by some methods.

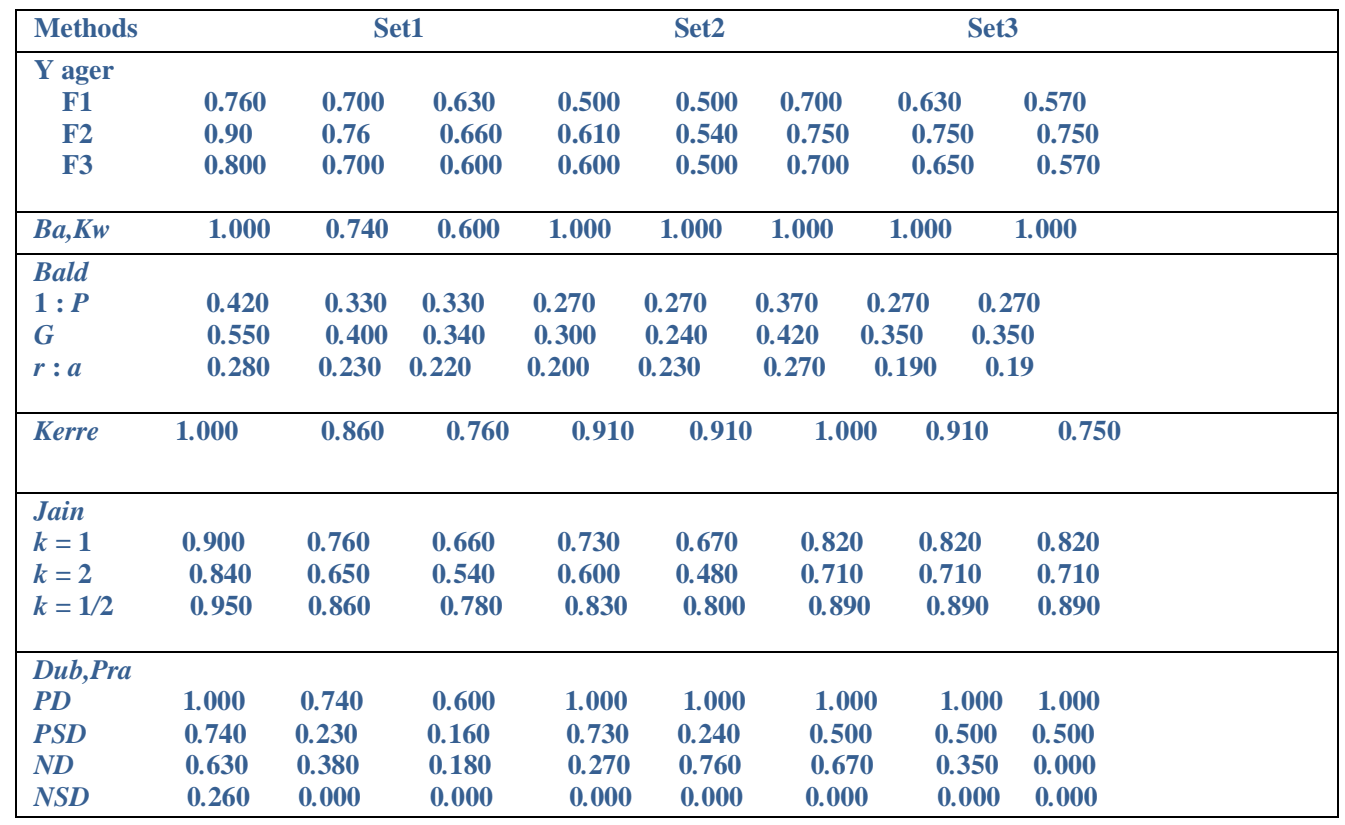




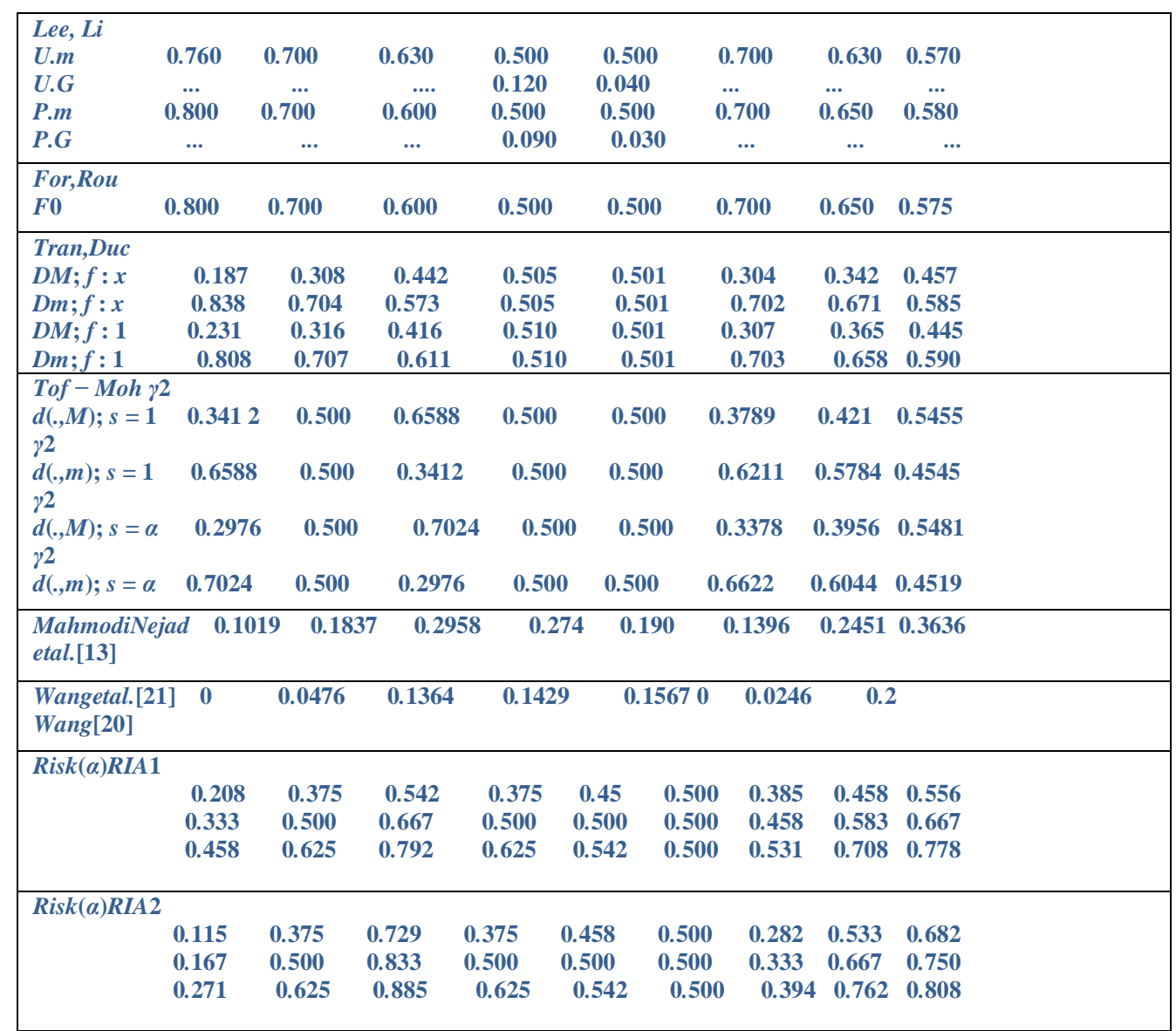

